\title{
Staged Surgery for Bronchobiliary Fistula and Incidental Finding of Mitral Valve Disease
}

\author{
Hazem Aljasem, Nizar Abbas, Younes Suliman, Ali Khaddam, Ahmad Al Khaddour* \\ Damascus University Hospital, Damascus, Syria \\ Email: *ahmedal@doctors.org.uk
}

How to cite this paper: Aljasem, H., Abbas, N., Suliman, Y., Khaddam, A. and Al Khaddour, A. (2016) Staged Surgery for Bronchobiliary Fistula and Incidental Finding of Mitral Valve Disease. World Journal of Cardiovascular Surgery, 6, 105-111. http://dx.doi.org/10.4236/wjcs.2016.69017

Received: August 10, 2016

Accepted: September 4, 2016

Published: September 7, 2016

Copyright $\odot 2016$ by authors and Scientific Research Publishing Inc. This work is licensed under the Creative Commons Attribution International License (CC BY 4.0).

http://creativecommons.org/licenses/by/4.0/

\begin{abstract}
Bronchobiliary fistula (BBF) is defined as abnormal passage between bronchial system and the bile tree which often manifests as presence of bile in the sputum (biloptysis). BBF is a serious complication which requires early diagnosis and well-planned management strategy to avoid the high morbidity and mortality rate. The hydatid cystic disease is still the leading cause of the BBF. In this paper we present a case of 32-year-old man with BBF who was incidentally found to have a mitral valve disease for which he underwent mitral valve replacement during his management course.
\end{abstract}

\section{Keywords}

Biloptysis, Mitral Valve Replacement, Hydatid Disease, Biliary Lithiasis, Cholecystitis, Endoscopic Retrograde Cholangiopancreatography (ERCP), Computed Tomography (CT), Magnetic Resonance

Cholangiography (MRC), Scintigraphy

\section{Introduction}

Bronchobiliary fistula (BBF) is an unusual disorder characterized by an abnormal communication between the biliary tract and the bronchial tree [1] which constitutes a major problem in thoracic surgery and requires aggressive treatment to decrease the mortality and morbidity rates [2]. It can occur as a congenital malformation, but most patients have a history of liver pathology that involves the lungs [3]. BBF can develop as a complication of hepatic surgery and interventions, or can be associated with various hepatobiliary diseases such as hydatid disease, hepatic abscesses, liver metastases, biliary lithiasis, and acute cholecystitis [1] [4]. The verification of the diagnosis and the demonstration of the site of communication along with underlying disease often require imaging tests. Endoscopic retrograde cholangiopancreatography (ERCP), computed tomography (CT), magnetic resonance cholangiography (MRC), and hepatobi- 
liary scintigraphy are the most commonly employed imaging modalities [4] [5]. Bronchobilary fistula is a rare but well-recognized complication of hydatid disease [6]. In this paper, we report a case of BBF in a man with hepatic hydatid cyst who was incidentally found to have a mitral valve disease for which he underwent mitral valve replacement through his treatment course.

\section{Case Presentation}

A 32-year-old man admitted to our hospital with biliptysis, dyspnea and intermittent chest pain for the last two months. His past medical history include laparotomy four months ago to excise a hepatic hydatid cyst. On clinical examination the patient was anemic. Examining the cardiovascular system revealed a 4/6 early systolic murmur at the heart apex. On lungs auscultation he had absent breath sounds at the base of right lung. The laboratory tests were within normal limits apart from the white blood cells which it was 14 (reference range $4.0-10.0 \times 10^{9} / \mathrm{L}$ ) with neutrophils 4.7 (reference range $1.5-7.0 \times 10^{9} / \mathrm{L}$ ), eosinophils 6 (reference range $0.1-0.5 \times 10^{9} / \mathrm{L}$ ) and mild anemia with hemoglobin $119 \mathrm{~g} / \mathrm{L}$ (reference range 116 - $156 \mathrm{~g} / \mathrm{L}$ ).

The chest X-ray revealed atelectasis at the right lung base with mild pleural effusion (as shown in Figure 1). The chest and abdominal CT scan with contrast revealed round atelectasis at the base of the right lung with mild pleural effusion, $1 \mathrm{~cm}$ right hillar and mediastenal lymph nodes. A fine fistula tract was detected between the biliary and

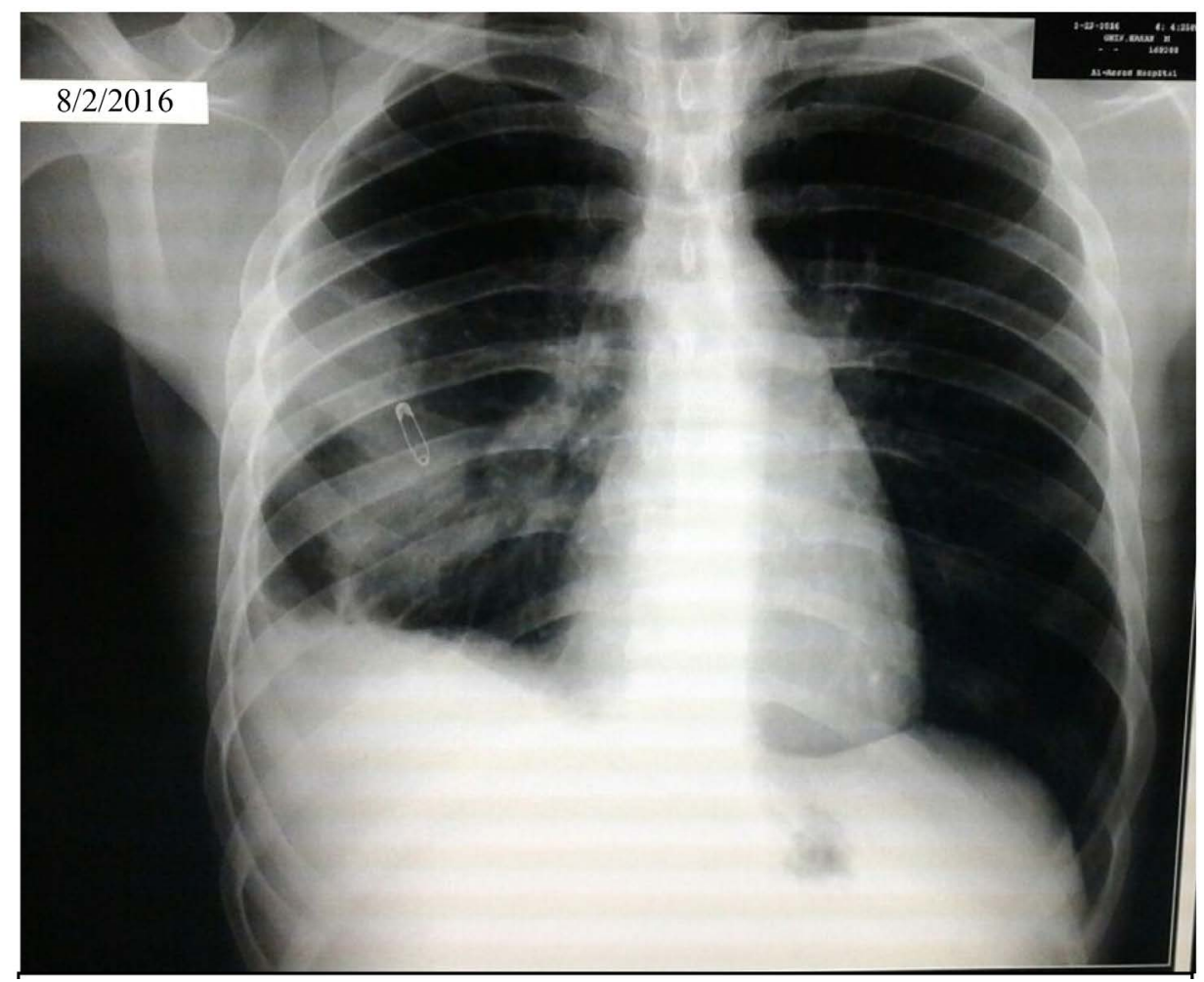

Figure 1. First admission CXR revealed atelectasis at the base of the right lung with mild pleural effusion. 
bronchial trees passing through the diaphragm (as shown in Figure 2). A transthoracic echocardiograph (TTE) was requested as part of routine work up for surgery which revealed a severe mitral stenosis and a moderate aortic regurgitation. The cardiologist was consulted who recommended for the patient to have a mitral valve replacement before closing the bronchobiliary fistula in order to reduce the risk associated with the procedure. However the patient was treated with a course of antibiotic. The biliptysis improved slightly and the patient was referred for cardiac surgery with consideration of mitral valve replacement.

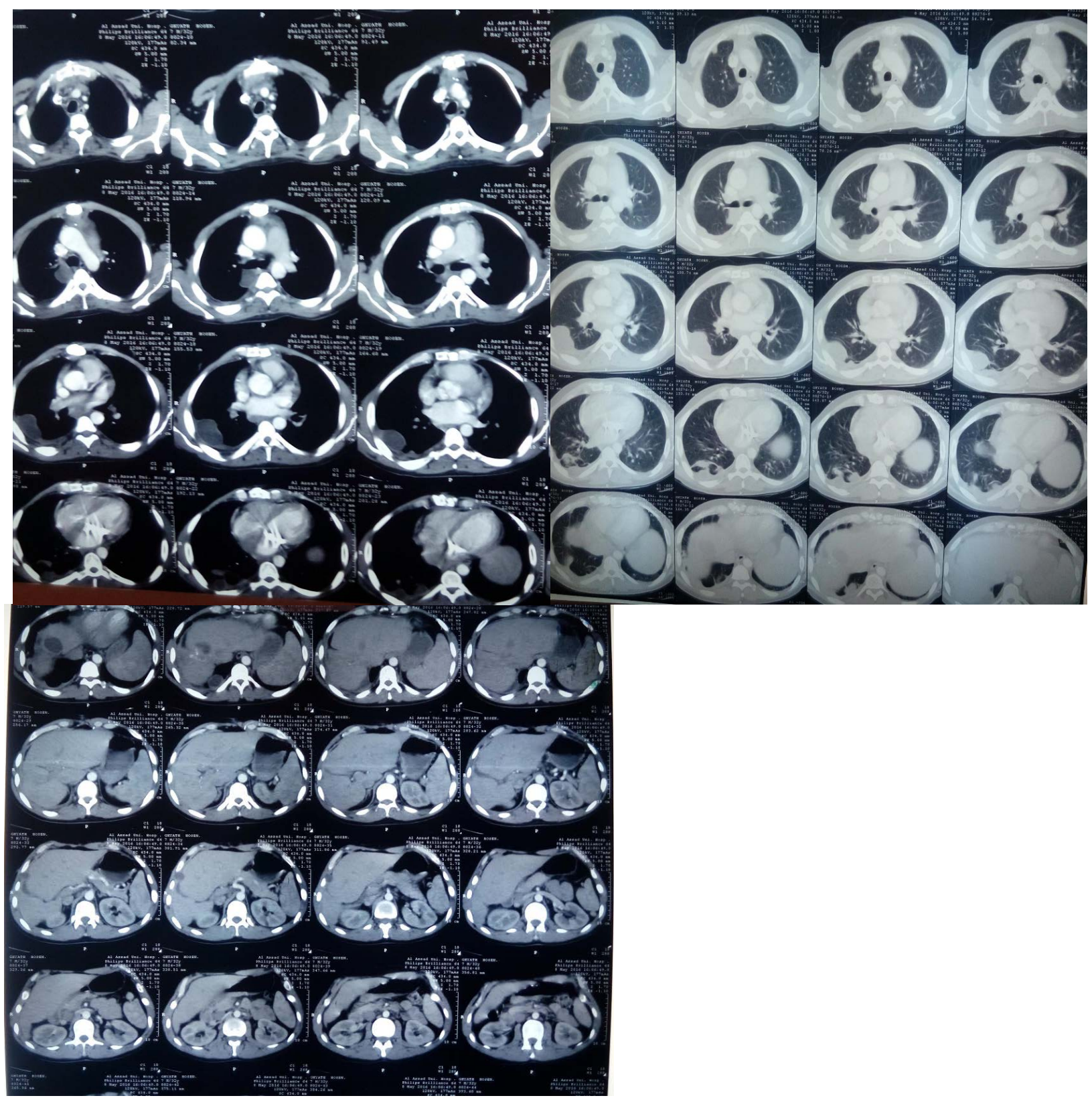

Figure 2. Post mitral valve replacement CT scan: chest CT showed cystic lesions at the base of right lower lobe of the lung: abdominal CT showed cystic lesions in the right lobe of the liver and the presence of the bronchobiliary fistula. 
Further transeosophageal echo confirmed severe mitral valve stenosis with mild aortic valve regurgitation and slightly dilated left ventricle.

The patient underwent heart surgery to replace his mitral valve. Under general anesthesia Median sternotomy was performed. The right pleura remained intact Systemic heparin administered and cardiopulmonary bypass instituted using bicaval cannulation for venous drainage and ascending aortic cannulation for arterial return. The patient was cooled to 33 degrees centigrade, aortic cross-clamp was applied and antegrade cold blood cardioplegia infused to achieve prompt cardiac arrest. The mitral valve was replaced with a mechanical valve size 29. The left atrium was closed with 40 nylon stitches. The cross-clamp was then released and the heart resumed in sinus rhythm.

Cardiopulmonary bypass was discontinued without complications.

Two chest drains were inserted, haemostasis secured and the chest closed using 7 single sternal wires. The soft tissues were approximated in two layers.

The skin was closed using monocryl (absorbable) suture. The patient had minimal mediastinal drainage postoperatively and he was transferred from intensive care unit to the ward on the second postoperative day. On five postoperative day the patient developed low grade temperature and he started to have biliptysis again. The endocarditis was excluded by echocardiography and the antibiotics was modified according to microbiology advice. biliptysis was persistent. He was discharged on 11th postoperative day on oral antibiotics, and long life warfarin. Two months later the patient readmitted to our department for his fistula operation. He had persistent biliptysis. The CXR has not changed. A new chest and abdominal CT scan with contrast revealed multiple cystic lesions at the base of right lung, Also there was a cystic lesion in the right lobe of the liver. The fistula was still present (as shown in Figure 2) a new transthoracic echo (TTE) confirmed that the mechanical mitral valve in a good position and is functioning well. The patient underwent ERCP (endoscopic retrograde cholangiopancreatography) with sphenectrectomy to secure a good biliary drainage prior to his fistula closure.

The patient underwent his second operation in order to close the bronchbiliary fistula under general anesthesia right thoracotomy was performed through the 7th intercostal space. There were a lot of adhesions between the lung, the chest wall and the diaphragm the right lower lobe was decorticated. Three hydatid cysts were found within the diaphragm and one in the right lower lobe of lung and one cyst in the right lobe of the liver. The bronchobiliary fistula was probed. The cysts were aspirated then the germinative membranes were resected. The fistula was debrided and excised completely.

Another cyst in the right lobe of the liver was resected. The diaphragm was plicated using prolene 1 continues suture. The thoracotomy was closed in layers over two chest drains and one small redovac drain inserted in supdiaphragmatic space. The soft tissues were approximated in three layers. The skin was closed using intradermal vicyle suture. The patient recovered well and discharged on 6th postoperative day without any complications. Postoperative CT scan was clear from cystic lesions and BBF (as shown in Figure 3). 


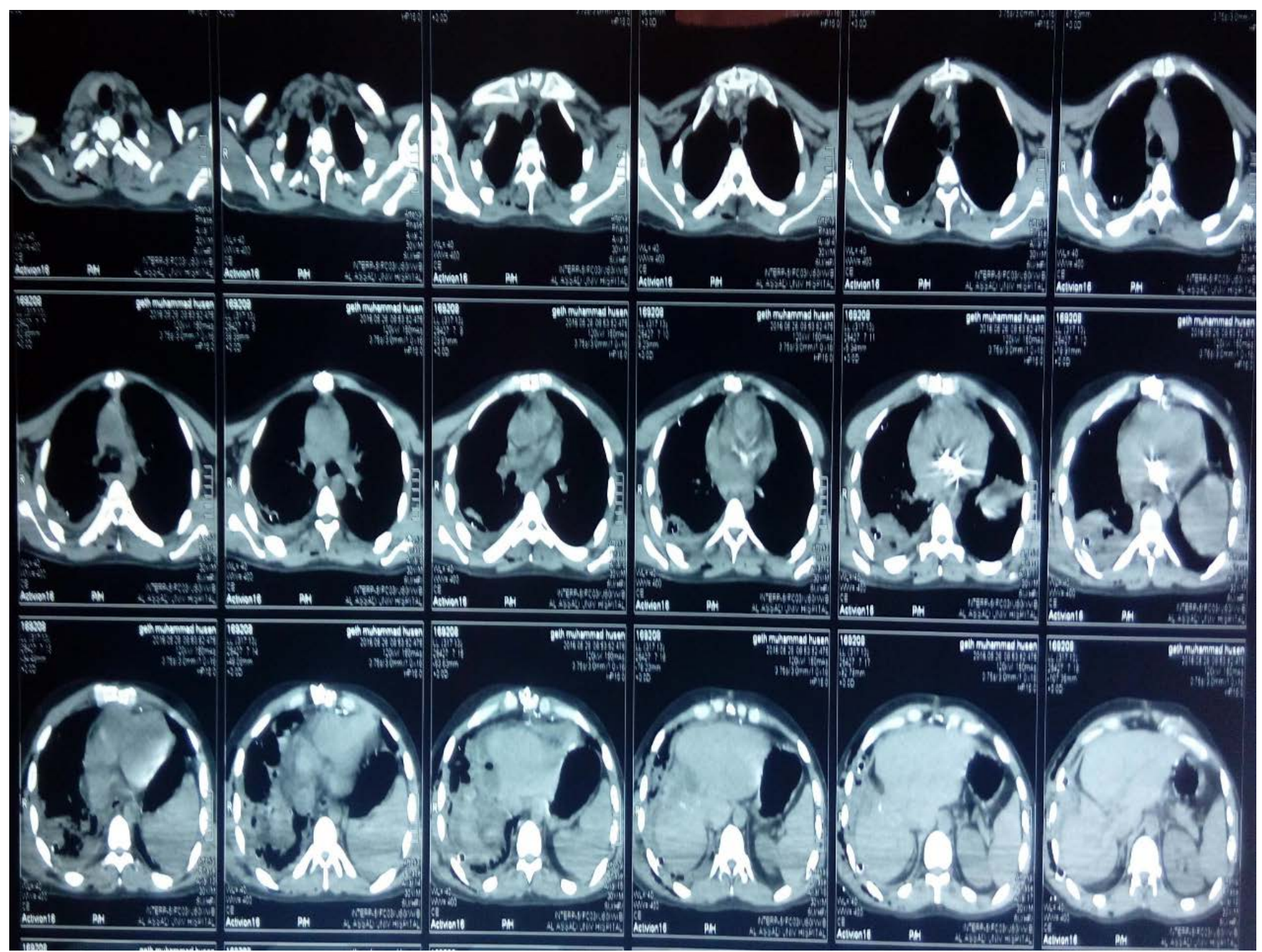

Figure 3. Post bronchobiliary fistula closure chest and abdominal CT scan: the cystic lesions and the fistula disappear.

\section{Discussion}

In this study we presented a 32-year-old man with BBF who incidentally found to have a mitral valve disease for which he underwent Mitral valve replacement during his management course.

$\mathrm{BBF}$ is an extremely a rare clinical condition. It was first reported by Peacock in 1850 [7], in this disorder a bile leak penetrates the diaphragm into the bronchial tree, more frequently on the right side [1].

Unlike congenital bronchobiliary fistula, acquired BBF is usually a complication of local infection, such as hydatid or amebic disease of the liver, hepatic abscess, trauma, obstruction of biliary tract and neoplasm [7]. Although hydatid disease is still the leading cause of BBF [2], BBF formation could be developed by several mechanisms, including an inflammatory reaction in the subdiaphragmatic space with subsequent ruptures into the bronchial system. Also it could be caused by any liver pathology which erodes the diaphragm leading to a communication between the bronchial tree and the 
biliary channels [2]. Bronchobiliary fistula may lead to a variety of pulmonary complications due to bile behaves as a strong irritant including recurrent chemical and bacterial pneumonitis, bronchiolitis, bronchiectasis, and mediastinitis [1]. A high mortality and morbidity rate $(12.2 \%)$ make $\mathrm{BBF}$ a serious complication which requires a wellplanned management strategy [2]. Clinical diagnosis is usually made by biliptysis which is prescribed as the presence of bile in the sputum and it is defined as pathognomonic bronchobiliary fistula [7]. Patients are persuaded that the fistula should be treated without delay following intense supportive therapy which includes proper antibiotics and high calorie intake. Management has traditionally been surgical resection, but recently embolization of the fistula [6] and stent placement to relieve common-bile-duct obstruction have been described [8]. When the cause of the fistula is echinococcal disease, open surgery may minimize the risk of cyst rupture complicated by peritoneal or pulmonary contamination [6]. The medical treatment includes somatostatin and its analogues are often used for treating BBF, because it reduces its secretion in the gastrointestinal tract. But, up till now, not a single case has been completely cured only with medical treatment [9].

Treatment of BBF requires the removal of distal obstruction, reduction of flow through the fistula, or excision of the fistula. The first therapeutic option in the management of $\mathrm{BBF}$ should be nonoperative radiologic and gastrointestinal interventions via external and internal stenting which reduce biliary obstruction. Operative approaches should be reserved only when interventional procedures fail, or in patients with advanced concurrent diseases [1]. Certainly whole treatment modalities fail if the obstruction in the biliary channel persists. Therefore, the first step following diagnosis is ensuring that the bile drains into duodenum without any obstruction. A management plan depends on the primary illness and the findings on initial assessment. In patients with a short life expectancy in whom BBF developed due to a malignant disease, conservative approaches as a biliary stent or biliary decompression with endoscopic sphincterotomy provide sufficient palliation [2] [10].

Recently, when resolution of a distal biliary obstruction was accomplished, non-surgical interventions via ERCP or PTC (Percutaneous transhepatic cholangogram) were successfully conducted [7].

In the past few years, histoacryl embolization under bronchoscopic guidance or the n-Butyl Cyanoacrylate via a bronchial approach were reported as new therapeutic methods [11] [12].

The open surgery should be the first choice when interventional techniques have failed or BBF secondary to tumors, biliary obstruction and trauma occurred [7]. On thoracotomy, the fistula tract has to be entirely exposed, which necessitates exploration of the subdiaphragmatic area and the dome of the liver. The tract has to be completely resected, and the liver pathology is repaired to ensure no bile leakage.

\section{Conclusion}

$\mathrm{BBF}$ is rare and serious complication which could cause a number of consequences and 
even leads to death. To avoid all that an early diagnosis and well planned management may alleviate the patient's sufferings. The outcome of bronchobiliary fistula secondary to hydatid disease is usually good if the condition is rapidly identified and treated. This study was approved by our ethical committee and it was presented at our hospital weekly scientific meeting. The patient gave the appropriate consent and agreed with the study.

\section{References}

[1] Karabulut, N., Çakmak, V. and Kiter, G. (2010) Confident Diagnosis of Bronchobiliary Fistula Using Contrast-Enhanced Magnetic Resonance Cholangiography. Korean Journal of Radiology, 11, 493-496. http://dx.doi.org/10.3348/kjr.2010.11.4.493

[2] Eryigit, H., Oztas, S., Urek, S., Olgac, G., Kurutepe, M. and Kutlu, C.A. (2007) Management of Acquired Bronchobiliary Fistula: 3 Case Reports and a Literature Review. Journal of Cardiothoracic Surgery, 2, 52. http://dx.doi.org/10.1186/1749-8090-2-52

[3] Singh, B., Moodley, J., Sheik-Gafoor, M.H., et al. (2002) Conservative Management of Thoracobiliary Fistula. Annals of Thoracic Surgery, 73, 1088-1091. http://dx.doi.org/10.1016/S0003-4975(02)03382-9

[4] Ragozzino, A., De Rosa, R., Galdiero, R., Maio, A. and Manes, G. (2005) Bronchobiliary Fistula Evaluated with Magnetic Resonance Imaging. Acta Radiologica, 46, 452-454. http://dx.doi.org/10.1080/02841850510021544

[5] Eryigit, H., Oztas, S., Urek, S., Olgac, G., Kurutepe, M. and Kutlu, C.A. (2007) Management of Acquired Bronchobiliary Fistula: 3 Case Reports and a Literature Review. Journal of Cardiothoracic Surgery, 2, 52. http://dx.doi.org/10.1186/1749-8090-2-52

[6] Rando, K., Harguindeguy, M. and Zunini, G. (2008) Echinococcal Disease with Bronchobiliary Fistula. Canadian Journal of Surgery, 51, E117-E118.

[7] Liao, G.-Q., Wang, H., Zhu, G.-Y., Zhu, K.-B., Lv, F.-X. and Tai, S. (2011) Management of Acquired Bronchobiliary Fistula: A Systematic Literature Review of 68 Cases Published in 30 Years. World Journal of Gastroenterology, 17, 3842-3849.

http://dx.doi.org/10.3748/wjg.v17.i33.3842

[8] Sharma, B.C., Agarwal, N., Garg, S., et al. (2006) Endoscopic Management of Liver Abscesses and Cysts that Communicate with Intrahepatic Bile Ducts. Endoscopy, 38, 249-253. http://dx.doi.org/10.1055/s-2005-921117

[9] Ong, M., Moozar, K. and Cohen, L.B. (2004) Octreotide in Bronchobiliary Fistula Management. Annals of Thoracic Surgery, 78, 1512-1513; Author Reply 1513. http://dx.doi.org/10.1016/j.athoracsur.2003.10.137

[10] Partrinou, V., Dougenis, D., Kritikos, N., et al. (2001) Treatment of Postoperative Bronchobiliary Fistula by Nasobiliary Drainage. Surgical Endoscopy, 15, 758.

http://dx.doi.org/10.1007/s004640040046

[11] Kim, J.H., Kim, M.D., Lee, Y.K., Hwang, S.G., Lee, J.H., Kim, E.K. and Jeong, H.C. (2008) Bronchobiliary Fistula Treated with Histoacryl Embolization under Bronchoscopic Guidance: A Case Report. Respiratory Medicine CME, 1, 164-168. http://dx.doi.org/10.1016/j.rmedc.2008.04.002

[12] Goldman, S.Y., Greben, C.R., Setton, A., McKinley, M.J., Axelrod, D.J., Charles, H.W. and Gandras, E.J. (2007) Bronchobiliary Fistula Successfully Treated with N-Butyl Cyanoacrylate via a Bronchial Approach. Journal of Vascular and Interventional Radiology, 18, 151155. http://dx.doi.org/10.1016/j.jvir.2006.10.012 
Submit or recommend next manuscript to SCIRP and we will provide best service for you:

Accepting pre-submission inquiries through Email, Facebook, LinkedIn, Twitter, etc. A wide selection of journals (inclusive of 9 subjects, more than 200 journals)

Providing 24-hour high-quality service

User-friendly online submission system

Fair and swift peer-review system

Efficient typesetting and proofreading procedure

Display of the result of downloads and visits, as well as the number of cited articles

Maximum dissemination of your research work

Submit your manuscript at: http://papersubmission.scirp.org/ 